\title{
EXISTENCE OF INTERPOLATING FUNCTIONS OF EXPONENTIAL TYPE
}

\author{
BY \\ RICHARD F. DEMAR(1)
}

1. Introduction. Let $K$ be the linear space of all entire functions of exponential type, and let $S$ be the linear space of all sequences $\left\{b_{n}\right\}$ of complex numbers satisfying $\lim \sup \left|b_{n}\right|^{1 / n}<\infty$. Let $T: K \rightarrow S$ be a linear transformation. Then these two problems are of interest.

1. The uniqueness problem: To find a subspace $C \subseteq K$ on which $T$ is oneto-one. Such a subspace is called a uniqueness class for $T$.

2. The interpolation problem: To determine for a given subspace $C \subseteq K$ the image $S_{c}$ of $C$ under the transformation $T$. If a sequence $\gamma \in S_{c}$, then $\gamma$ is said to be admissible for the transformation $T$ and the class $C$. A function $f$ such that $T(f)=\gamma$ said to interpolate $\gamma$ relative to the transformation $T$.

In this paper, the method of associated functions [3] is used to establish a theorem which gives, for a general class of linear transformations $T$, a necessary and sufficient condition that a sequence $\gamma$ be admissible for $T$ and a corresponding uniqueness class $C \subseteq K$. This theorem is then applied to a number of specific transformations $T$. In some cases, other transformations, to which this theorem does not apply, yield admissibility results by the same method. Two such examples are given in $\$ 5$. The question of convergence or summability of a series $\sum b_{n} p_{n}(z)$ for a sequence $\left\{p_{n}(z)\right\}$ of polynomials satisfying a certain generating relation is discussed in $\$ 6$.

2. Background and notation. Let $f(z)=\sum a_{n} z^{n} / n$ ! belong to $K$. The Borel transform of $f$ is the function $F(w)$ defined by $\sum a_{n} w^{-n-1}$ and its analytic continuation. The convex hull of the set of singularities of $F$ is called the conjugate indicator diagram of $f$ and is denoted by $D(f)$. The growth function of $f$ in $K$ is given by

$$
h(\theta, f)=\limsup _{r \rightarrow \infty} \frac{\log f\left(r e^{i \theta}\right)}{r} .
$$

The supporting function of a closed set $G$ in the plane is defined by $k(\theta, G)$ $=\max _{z \in G} \mathscr{R}\left(z e^{-i \theta}\right)$ (finite or infinite). If $G$ is convex, then a closed set $G_{1}$ is con-

Preserited to the Society, January 24, 1962; received by the editors November 10, 1961.

(1) This paper is part of the author's doctoral dissertation at the University of Wisconsin. The author is indebted to Professor R.C. Buck who suggested the problem and directed the study. 
tained in $G$ if and only if $k\left(\theta, G_{1}\right) \leqq k(\theta, G)$ for all $\theta$. Pólya [7] proved the important result that for $f$ in $K, h(\theta, f)=k(-\theta, D(f))$. For any connected set $G$ in the plane, let $K[G]$ denote the set of all functions $f$ in $K$ such that $D(f) \subseteq G$. In case $G$ is a rectangle $\{x+i y|| x|\leqq a| y \mid, \leqq c\}$, we denote $K[G]$ by $K[a, c]$.

The linear transformations $T: K \rightarrow S$ dealt with will be those given by sequences $\left\{\mathscr{L}_{n}\right\}$ of linear functionals having a representation

$$
\mathscr{L}_{n}(f)=\frac{1}{2 \pi i} \int_{\Gamma} g_{n}(\zeta) F(\zeta) d \zeta
$$

for some sequence $\left\{g_{n}\right\}, g_{n} \in K$, where $F$ is the Borel transform of $f$ and $\Gamma$ is a simple contour enclosing $D(f)$. The functions $g_{n}$ are called generating functions. If $f(z)=e^{z t}$, then $\mathscr{L}_{n}(f)=g_{n}(t)$ [2]. Our main concern will be with transformations $T$ for which the generating functions have the form $[W(\zeta)]^{n}$ for some function $W(\zeta)$ such that $W(0)=0$ and $W^{\prime}(0) \neq 0$, in which case 2.1 becomes

$$
\mathscr{L}_{n}(f)=\frac{1}{2 \pi i} \int_{\Gamma}[W(\zeta)]^{n} F(\zeta) d \zeta
$$

Many sequences of linear functionals which have been studied are of this form; e.g., the Taylor functionals $(W(\zeta)=\zeta)$ and the Newton functionals $\left(W(\zeta)=e^{\zeta}-1\right)$.

Certain sets associated with the function $W(\zeta)$ will be used. Let $W$ be regular and univalent on an open, connected set $\Omega_{\zeta}$ containing the origin. Let the image of $\Omega_{\zeta}$ under $w=W(\zeta)$ be $\Omega_{w}$ and let $\Omega_{w}^{*}$ be the star of $\Omega_{w}$; i.e., $\Omega_{w}^{*}=\left\{w \mid \lambda w \in \Omega_{w}\right.$ for all $\lambda$ such that $0 \leqq \lambda \leqq 1\}$. Let $\Omega_{\zeta}^{*} \subseteq \Omega_{\zeta}$ be the image of $\Omega_{w}^{*}$ under $\zeta=g(w)$, the inverse of $W(\zeta)$. The largest disk, $|w|<\rho_{0}$, contained in $\Omega_{w}$ will be denoted by $\Delta_{w}$ and its image under $\zeta=g(w)$ by $\Delta_{\zeta}$. Associated with a sequence $\left\{b_{n}\right\}$ in $S$ is a function defined by $\sum b_{n} z^{n}$ and its analytic continuation. This will be denoted by $b(z) . A^{\prime}$ will denote the complement of $A$, and $1 / A$ the image of $A$ under the map $S=1 / t$. This notation follows that of Boas and Buck [1]. Summations without limits shown indicate sums from 0 to $\infty$.

3. Main theorem. For a linear transformation $T$ given by a sequence of linear functionals $\left\{\mathscr{L}_{n}\right\}$ having a representation 2.2, Buck [3] gave a necessary condition for a sequence $\left\{b_{n}\right\}$ to be admissible for $T$ and a class $K\left[C_{\zeta}\right] \subseteq K\left[\Omega_{\zeta}^{*}\right]$ of functions. The main theorem of this paper is that if $C_{\zeta}$ is a convex set containing the origin, then the condition is also sufficient.

THEOREM 3.1. Let $T=\left\{\mathscr{L}_{n}\right\}$ have a representation 2.2. Let $C_{\zeta}$ be an open convex subset of $\Omega_{\zeta}$ containing the origin. Then a necessary and sufficient condition that a sequence $\left\{b_{n}\right\}$ in $S$ be admissible for the transformation $T$ and the class $K\left[C_{\xi}\right]$ is that $b(z)$ be regular on $1 / C_{w}^{\prime}$ where $C_{w}$ is the image of $C_{\zeta}$ under the map $w=W(\zeta)$. 
Proof. For necessity, see Buck [3]. For sufficiency, let $\left\{b_{n}\right\}$ in $S$ be such that $b(z)$ is analytic on $1 / C_{w}^{\prime}$. Let $f$ be defined by

$$
f(z)=\frac{1}{2 \pi i} \int_{E} \frac{b(t)}{t} e^{z g(1 / t)} d t
$$

where $g(w)$ is the inverse of $W(\zeta)$ and $E$ is a simple contour contained in the region of regularity of $b(t)$ and enclosing the closed set $1 / C_{w}^{\prime}$. Then by the continuity and linearity of the functionals $\mathscr{L}_{n}$,

$$
\mathscr{L}_{n}(f)=\frac{1}{2 \pi i} \int_{E} \frac{b(t)}{t} \mathscr{L}_{n}\left(e^{z g(1 / t)} d t\right.
$$

But $\mathscr{L}_{n}\left(e^{2 \zeta}\right)=[W(\zeta)]^{n}$; so $\mathscr{L}_{n}\left(e^{2 g(1 / t)}\right)=[W(g(1 / t))]^{n}=1 / t^{n}$. Thus, since $E$ encloses the origin,

$$
\mathscr{L}_{n}(f)=\frac{1}{2 \pi i} \int_{E} \frac{b(t)}{t^{n+1}}=\frac{b^{(n)}(0)}{n !}=b_{n}
$$

Also, since $E$ encloses $1 / C_{w}^{\prime}, E \subseteq 1 / C_{w}$; so its image $\Gamma$ in the $\zeta$-plane under $\zeta=g(1 / t)$ is contained in $C_{\zeta}$. Then from the definition of $f$, for any $z=r e^{i \theta}$,

so that

$$
\begin{aligned}
|f(z)| & \leqq \max _{t \in E} e^{z g(1 / t)} \frac{1}{2 \pi} \int_{E}\left|\frac{b(t)}{t}\right||d t| \\
& =M \max _{\zeta \in \Gamma}\left|e^{z \zeta}\right|
\end{aligned}
$$

$$
\begin{aligned}
f\left(r e^{i \theta}\right) & \leqq M \max _{\zeta \in \Gamma}\left|e^{r e^{i \theta}}\right| \\
& \leqq M \exp \left[r \max _{\zeta \in \Gamma} \mathscr{R}\left(\zeta e^{i \theta}\right)\right] .
\end{aligned}
$$

Thus, taking principal values of logarithms,

so

$$
\log \left|f\left(r e^{i \theta}\right)\right| \leqq r \max _{\zeta \in \Gamma} \mathscr{R}\left(\zeta e^{i \theta}\right)+\log M
$$

$$
\limsup _{r \rightarrow \infty} \frac{\log f\left(r e^{i \theta}\right)}{r} \leqq \max _{\zeta \in \Gamma} \mathscr{R}\left(\zeta e^{i \theta}\right),
$$

or $h(\theta, f) \leqq k(-\theta, \Gamma)$. Therefore $k(\theta, D(f)) \leqq k(\theta, \Gamma)$; so $D(f)$ is contained in the convex hull of $\Gamma$ which is contained in $C_{\zeta}$ since $C_{\zeta}$ is convex. Therefore $f \in K\left[C_{\zeta}\right]$. Q.E.D.

COROLlaRY 3.2. If, for the transformation $T$ of Theorem 3.1, the set $\Delta_{\zeta}$ is convex, then a sequence $\left\{b_{n}\right\}$ is admissible for $T$ and $K\left[\Delta_{\zeta}\right]$ if and only if $\lim \sup \left|b_{n}\right|^{1 / n}<\rho_{0}$. 
4. Application to specific transformations. In this section, the main theorem is applied to the linear transformations given by the sequences of generalized difference functionals, Abel functionals, and generalized Newton functionals.

A. Generalized Difference fUnCtionals. These functionals are given by $\mathscr{L}_{n}(f)=\Delta^{n} f(\beta n)=\sum_{k=0}^{n}(-1)^{n+k} C_{n, k} f(\beta n+k)$. The generating functions are $g_{n}(\zeta)=e^{\beta n \zeta}\left(e^{\zeta}-1\right)^{n}$; so $W(\zeta)=e^{\beta \zeta}\left(e^{\zeta}-1\right)$. Uniqueness results have been given only for $\beta \geqq 0$ and $\beta=-1 / 2$; so we shall give admissibility results only for these values of $\beta$. Results for other values will be given in a later paper. For $\beta>0$, Buck [2] showed that $\Omega_{\zeta}$ can be taken as the region containing the origin and bounded by the curve $x=\log (\sin \beta y)-\log (\sin (\beta+1) y)$ where $\zeta=x+i y$. The set $\Omega_{w}$ is the $w$ plane cut from $w_{0}=-\beta^{\beta}(\beta+1)^{-(\beta+1)}$ to $-\infty$ along the negative real axis. From Theorem 3.1, we have

Corollary 4.1. Let $\mathscr{L}_{n}(f)=\Delta^{n} f(\beta n), \beta>0$, and let $\Omega_{\zeta}$ be the set given above. A sequence $\left\{b_{n}\right\}$ is admissible for $T=\left\{\mathscr{L}_{n}\right\}$ and $K\left[\Omega_{\zeta}\right]$ if and only if $b(z)$ is regular on the interval $-(\beta+1)^{\beta+1} \beta^{-\beta} \leqq x \leqq 0$ of the real axis.

For example, if $\beta=1 / 2$, this interval is approximately $[-1.60,0]$; if $\beta=1$, it is $[-4,0]$.

If $\beta=0$, the functionals are the familiar Newton functionals $\Delta^{n} f(0)$. Then $\Omega_{\zeta}$ can be taken as the strip $\{x+i y|| y \mid<\pi\}$ and $\Omega_{w}$ is then the $w$-plane cut from -1 to $-\infty$ along the negative real axis [2].

Corollary 4.2. A sequence $\left\{b_{n}\right\}$ in $S$ is admissible for the Newton functionals and $K[a, c], c<\pi$, if and only if $b(z)$ is regular on the interval $[-1,0]$ of the real axis.

This result was obtained earlier by Buck [4].

For these functionals, the set $\Delta_{\zeta}$ is the set containing the origin and bounded by the curve $x=\log (2 \cos y)$ and $\Delta_{w}$ is the disk $|w|<1$ [2]. Using the result that the interpolation series $\sum C_{z, n} \Delta^{n} f(0)$, where $C_{z, n}=z(z-1) \cdots(z-n+1) / n$ !, converges to $f$ for any $f \in K\left[\Delta_{\zeta}\right]$ and Corollary 3.2, we obtain

CoRollary 4.3. The series $\sum b_{n} C_{z, n}$ converges to a function $f$ in $K\left[\Delta_{\zeta}\right]$ if and only if $\lim \sup \left|b_{n}\right|^{1 / n}<1$.

If $\beta=-1 / 2$, the functionals are called Stirling functionals. In this case, $\Omega_{\zeta}$ can again be taken as the strip $|y|<\pi$ and then $\Omega_{w}$ is the $w$-plane cut from $2 i$ to $i \infty$ and from $-2 i$ to $-i \infty$ along the imaginary axis. Then $\Delta_{\zeta}$ is the region consisting of all $\zeta$ satisfying $|\sinh \zeta / 2|<1[2]$.

Corollary 4.4. A sequence $\left\{b_{n}\right\}$ in $S$ is admissible for the Stirling functionals and $K[a, c], c<\pi$ if and only if $b(z)$ is regular on the interval $[-(1 / 2) i,(1 / 2) i]$ of the imaginary axis. 
Corollary 4.5. A sequence $\left\{b_{n}\right\}$ in $S$ is admissible for the Stirling functionals and $K\left[\Delta_{\zeta}\right]$ if and only if lim sup $\left|b_{n}\right|^{1 / n}<2$.

B. Generalized Newton functionals. Given a complex number $A$, the generalized Newton functionals relative to $A$ are given by

$$
\mathscr{L}_{n}^{A}(f)=\left(-e^{A}\right)^{n} \sum_{k=0}^{n} C_{n, k}\left(-e^{A}\right)^{-k} f(k)
$$

For these, $W(\zeta)=e^{\zeta}-e^{A}$. If $A=0$, they are the ordinary Newton functionals. We take $A$ to be a positive real number. For $f$ in $K$, let $f_{0}(z)=e^{-A z} f(z)$; then $D\left(f_{0}\right)$ is the set $D(f)$ translated a distance $A$ to the left; i.e., $z \in D(f)$ if and only if $(z-A) \in D\left(f_{0}\right)\left[1\right.$, p. 14]. Also $\mathscr{L}_{n}^{A}(f)=e^{A n} \Delta^{n} f_{0}(0)$.

THEOREM 4.6. A sequence $\left\{b_{n}\right\}$ in: $S$ is admissible for a sequence of generalized Newton functionals $\left\{\mathscr{L}_{n}^{A}(f)\right\}, A$ a positive real number, and $K[a, c], c<\pi$, if and only if $b(z)$ is regular on the interval $\left[-e^{-A}, 0\right]$ of the real axis.

Proof. Let $f \in K[a, c], c<\pi$, and let $f_{0}(z)=e^{-A z} f(z)$. Since $f_{0} \in K\left[a^{\prime}, c\right], c<\pi$, $\sum \Delta^{n} f_{0}(0) z^{n}$ is regular on the interval $[-1,0]$ by Corollary 4.2 . Thus, since $\Delta^{n} f_{0}(0)=e^{-A n} \mathscr{L}_{n}^{A}(f), \sum e^{-A n} \mathscr{L}_{n}^{A}(f) z^{n}$ is regular on $\left[-e^{-A}, 0\right]$.

Conversely, let $b(z)=\sum b_{n} z^{n}$ be regular on $\left[-e^{-A}, 0\right]$. Then $\sum e^{-A n} b_{n} z^{n}$ is regular on $[-1,0]$, so by Corollary 4.2 , there exists $f_{0} \in K\left[a^{\prime}, c\right], c<\pi$, such that $\Delta^{n} f_{0}(0)=e^{-A n} b_{n} ; n=0,1,2, \cdots$. Let $f(z)=e^{A z} f_{0}(z)$. Then $f \in K[a, c], c<\pi$, and $\mathscr{L}_{n}^{A}(f)=b_{n} ; n=0,1,2, \cdots$. Q.E.D.

COROLlary 4.7. For any sequence $\left\{b_{n}\right\}$ in $S$, there exists a real number $A$ such that $\left\{b_{n}\right\}$ is admissible for $T=\left\{\mathscr{L}_{n}^{A}\right\}$ and $K[a, c], c<\pi$.

In a similar way, using Corollary 4.3 , it can be proved that for $A$ sufficiently large, $\left\{b_{n}\right\}$ is admissible for $T=\left\{\mathscr{L}_{n}^{A}\right\}$ and $K[a, c], c<\pi / 2$.

C. Abel functionals. These functionals are' given by $\mathscr{L}_{n}(f)=f^{(n)}(n)$; so $W(\zeta)=\zeta e^{\zeta}$. The set $\Omega_{\zeta}$ can be taken as the region containing the origin and bounded by the curve $\rho=(\pi-|\phi|) \csc |\phi|$ where $\zeta=\rho e^{i \phi}[2]$. This curve opens to the right, cuts the real axis at -1 , and has the lines $y= \pm \pi$, as asymptotes. Then $\Omega_{w}$ is the $w$-plane cut from $-e^{-1}$ to $-\infty$. The set $\Delta_{w}$ is the disk $|w|<e^{-1}$ and $\Delta_{\zeta}$ is the convex set determined by $\left|\zeta e^{1+\zeta}\right|<1$.

Corollary 4.8. A sequence $\left\{b_{n}\right\}$ in $S$ is admissible for $T=\left\{f^{(n)}(n)\right\}$ and $K\left[\Omega_{\zeta}\right]$ if and only if $b(z)$ is regular on the interval $[-e, 0]$ of the real axis.

Corollary 4.9. A sequence $\left\{b_{n}\right\}$ in $S$ is admissible for $T=\left\{f^{(n)}(n)\right\}$ and $K\left[\Delta_{\zeta}\right]$ if and only if $\lim \sup \left|b_{n}\right|^{1 / n}<e^{-1}$.

From Corollary 4.2 and Corollary 4.9, we obtain

Corollary 4.10. A sequence $\left\{b_{n}\right\}$ in $S$ is admissible for the Newton functionals and $K[a, c], c<\pi$, if and only if the sequence $\left\{e^{-n} b_{n}\right\}$ is admissible for the Abel functionals and the corresponding uniqueness class $K\left[\Omega_{\zeta}\right]$. 
This implies that if $f \in K\left[\Omega_{\zeta}\right]$ for the Abel functionals, then there exists a function $f^{*} \in K[a, c], c<\pi$, such that $f^{(n)}(n)=\Delta^{n} f^{*}(0) ; n=0,1,2, \cdots$.

5. Further admissibility results. The proof of the main theorem depended on the fact that the generating functions $g_{n}$ of the functionals were of the form $W^{n}$ for some function $W$. In certain cases, the generating functions of a transformation may not be of this form but the present methods still apply. We give two examples-the Newton-Gauss transformation given by $\mathscr{L}_{2 n}(f)=\Delta^{2 n} f(-n)$ and $\mathscr{L}_{2 n+1}(f)=\Delta^{2 n+1} f(-n)$, and the Lidstone transformation given by $\mathscr{L}_{2 n}(f)$ $=f^{(2 n)}(0)$ and $\mathscr{L}_{2 n+1}(f)=f^{(2 n)}(1)$. The method used follows that of Buck $[2 ; 5]$.

For the Newton-Gauss functionals, $g_{2 n}(\zeta)=\left(e^{\zeta / 2}-e^{-\zeta / 2}\right)^{2 n}$ and $g_{2 n+1(\zeta)}$ $=e^{\zeta / 2}\left(e^{\zeta / 2}-e^{\zeta / 2}\right)^{2 n+1}$. Let $W(\zeta)=e^{\zeta / 2}-e^{-\zeta / 2}$, so that $g_{2 n}(\zeta)=[W(\zeta)]^{2 n}$ and $g_{2 n+1}(\zeta)=e^{\zeta / 2}[W(\zeta)]^{2 n+1}$. Let $\Omega_{\zeta}$ and $\Omega_{w}$ be the same as for the Stirling functionals.

THEOREM 5.1. A sequence $\left\{b_{n}\right\}$ in $S$ is admissible for the Newton-Gauss functionals and $K[a, c], c<\pi$, if and only if $b(z)$ is regular on the interval $[-(1 / 2) i,(1 / 2) i]$ of the imaginary axis.

Proof. Given $f \in K[a, c], c<\pi$, define a function $b(z)$ by

$$
b(z)=\frac{1}{2 \pi i} \int_{\Gamma} \frac{1+e^{\zeta / 2} W(\zeta) z}{1-[W(\zeta)]^{2} z^{2}} F(\zeta) d \zeta
$$

where $\Gamma$ is any simple contour in $\Omega_{\zeta}$ that encloses $D(f)$ and the origin. Then

$$
\begin{aligned}
\frac{b^{(2 n)}(0)}{(2 n) !} & =\frac{1}{2 \pi i} \int_{\Gamma}[W(\zeta)]^{2 n} F(\zeta) d \zeta=\mathscr{L}_{2 n}(f), \\
\frac{b^{(2 n+1)}(0)}{(2 n+1) !} & =\frac{1}{2 \pi i} \int_{\Gamma} e^{\zeta / 2} W(\zeta)^{2 n+1} F(\zeta) d \zeta=\mathscr{L}_{2 n+1}(f),
\end{aligned}
$$

so that $b(z)=\sum \mathscr{L}_{n}(f) z^{n}$ in some neighborhood of the origin. From its integral representation, $b$ is regular at least for all $z$ such that for all $\zeta$ on $\Gamma, z^{2} \neq[W(\zeta)]^{-2}$; i.e., $z \neq \pm[W(\zeta)]^{-1}$. Since $\Omega_{\zeta}$ is symmetric with respect to the origin, $\Gamma$ can be taken symmetric with respect to the origin. Then $E$, the image of $\Gamma$ under $t=[W(\zeta)]^{-1}$, is symmetric with respect to the origin since $W$ is an odd function. Thus, $b$ is regular for all $z$ not lying on $E$, since $z \neq[W(\zeta)]^{-1}$ implies $z \neq \pm[W(\zeta)]^{-1}$ Since $\Gamma \subseteq \Omega_{\zeta}$ and encloses the origin, its image under $w=W(\zeta)$ is contained in $\Omega_{w}$ and encloses the origin, so that $E$ encloses $1 / \Omega_{w}^{\prime}$ which is the interval $[-(1 / 2) i,(1 / 2) i]$. Therefore $b$ is regular on this interval.

Conversely, let $\left\{b_{n}\right\}$ in $S$ be such that $b(z)$ is regular on $[-(1 / 2) i,(1 / 2) i]$. Let $\left\{p_{n}(z)\right\}$ be the sequence of polynomials determined by the formal relation,

$$
e^{z \zeta}=\sum p_{n}(z) g_{n}(\zeta)
$$


Letting

$$
\begin{aligned}
& H_{1}(z, \zeta)=\sum p_{2 n}(z)[W(\zeta)]^{2 n} \\
& H_{2}(z, \zeta)=\sum p_{2 n+1}(z)[W(\zeta)]^{2 n+1},
\end{aligned}
$$

define a function $f$ by

$$
f(z)=\frac{1}{2 \pi i} \int_{E} \frac{b(t)}{t}\left[H_{1}(z, g(1 / t))+H_{2}(z, g(1 / t))\right] d t
$$

where $g$ is the inverse of the function $W$, and $E$ is a simple contour symmetric with respect to the origin enclosing $[-(1 / 2) i,(1 / 2) i]$ and contained in the region of regularity of $b$. Then, by the orthonormality of $\left\{p_{n}(z)\right\}$ with respect to $\left\{\mathscr{L}_{n}\right\}$, we have

$$
\begin{array}{rlrl}
\mathscr{L}_{n}(f) & =\frac{1}{2 \pi i} \int_{E} \frac{b(t)}{t} \mathscr{L}_{n}\left[H_{1}(z, g(1 / t))+H_{2}(z, g(1 / t))\right] d t, & \\
& =\frac{1}{2 \pi i} \int_{E} \frac{b(t)}{t^{n+1}} d t=\frac{b^{(n)}(0)}{n !}=b_{n}, \quad n=0,1,2, \cdots
\end{array}
$$

It remains to show $D(f) \subseteq \Omega_{\zeta}$. From the evenness of $H_{1}$ and the oddness of $\mathrm{H}_{2}$, one obtains

and

$$
\begin{aligned}
H_{1}(z, \zeta) & =\frac{\cosh [(z-1 / 2) \zeta]}{\cosh (1 / 2) \zeta} \text { and } H_{2}(z, \zeta)=\frac{\sinh z \zeta}{\cosh (1 / 2) \zeta} \\
f(z) & =\frac{1}{2 \pi i} \int_{E} \frac{b(t)}{t} \frac{\cosh [(z-1 / 2) g(1 / t)]+\sinh [z g(1 / t)]}{\cosh (1 / 2) g(1 / t)} d t
\end{aligned}
$$

$$
|f(z)| \leqq O(1) \max _{t \in E}\left|\frac{\cosh [(z-1 / 2) g(1 / t)]+\sinh [z g(1 / t)]}{\cosh (1 / 2) g(1 / t)}\right| .
$$

Let $\Gamma$ be the image of $E$ under $\zeta=g(1 / t)$. Then

$$
\begin{aligned}
|f(z)| & \leqq O(1) \max _{\zeta \in \Gamma}\left|\frac{\cosh (z-1 / 2) \zeta+\sinh z \zeta}{\cosh (1 / 2) \zeta}\right| \\
& =O(1) \max _{\zeta \in \Gamma}|\cosh (z-1 / 2) \zeta+\sinh z \zeta| \\
& =O(1) \max _{\zeta \in \Gamma}\left|e^{z \zeta} e^{-\zeta / 2}+e^{-z \zeta} e^{\zeta / 2}+e^{z \zeta}-e^{-z \zeta}\right| \\
& =O(1) \max _{ \pm \zeta \in \Gamma}\left|e^{z \zeta}\right| .
\end{aligned}
$$

But $E$ is symmetric with respect to the origin, and $g(1 / t)=2 \sinh ^{-1}(1 / 2 t)$ is an odd function; so $\Gamma$ is symmetric with respect to the origin. Therefore $\max _{ \pm \zeta \in \Gamma}\left|e^{z \zeta}\right|=\max _{\zeta \in \Gamma}\left|e^{z \zeta}\right|$; so just as in the proof of Theorem $3.1, D(f) \subseteq \Omega_{\zeta}$. Thus $\left\{b_{n}\right\}$ is admissible for $\left\{\mathscr{L}_{n}\right\}$ and $K[a, c], c<\pi$. Q.E.D.

For the Lidstone functionals, $g_{2 n}(\zeta)=\zeta^{2 n}$ and $g_{2 n+1}(\zeta)=e^{\zeta \zeta^{2 n}}$, if $\Omega_{\zeta}$ is taken 
as the $\zeta$ plane cut from $i \pi$ to $i \infty$ and from $-i \pi$ to $-i \infty$ along the imaginary axis, then $K\left[\Omega_{\zeta}\right]$ is a uniqueness class for this transformation [2].

THEOREM 5.2. If $\left\{\mathscr{L}_{n}\right\}$ is the sequence of Lidstone functionals, and $\Omega_{\zeta}$ is the set given above, then for $f \in K\left[\Omega_{\zeta}\right]$, the function $b(z)=\sum \mathscr{L}_{n}(f) z^{n}$ is regular at the origin and can be continued to the interval $[-(1 / \pi) i,(1 / \pi) i]$ of the imaginary axis.

Proof. Let

$$
b(z)=\frac{1}{2 \pi i} \int_{\Gamma} \frac{1+z e^{\zeta}}{1-z^{2 \zeta^{2}}} F(\zeta) d \zeta
$$

where $\Gamma$ is a simple contour contained in $\Omega_{\zeta}$ and enclosing $D(f)$. Then, as in the proof of Theorem 5.1,

$$
f^{(2 n)}(0)=\frac{b^{(2 n)}(0)}{(2 n) !} \text { and } f^{(2 n)}(1)=\frac{b^{(2 n+1)}(0)}{(2 n+1) !} ;
$$

so $b(z)=\sum \mathscr{L}_{n}(f) z^{n}$ in some neighborhood of the origin. From its definition, $b$ is regular for every $z$ such that for all $\zeta$ on $\Gamma, \neq z \pm 1 / \zeta$. Since $\Omega_{\zeta}$ is symmetric with respect to the origin, $\Gamma$ can be taken symmetric with respect to the origin, so that if for all $\zeta$ on $\Gamma, z \neq 1 / \zeta$, then for all $\zeta$ on $\Gamma, z \neq \pm 1 / \zeta$. Thus $b$ is regular on the set enclosed by the image of $\Gamma$ in the $1 / \zeta$-plane. In particular, this includes the interval $[-(1 / \pi) i,(1 / \pi) i]$ of the imaginary axis. Q.E.D.

Because of the nonconvexity of $\Omega_{\zeta}$ for the Lidstone functionals, we were able to give only a necessary condition that a sequence $\left\{b_{n}\right\}$ be admissible for $\left\{\mathscr{L}_{n}\right\}$ and $K\left[\Omega_{\zeta}\right]$. To give a necessary and sufficient condition, the class of functions $K\left[C_{\zeta}\right]$ must be restricted to convex sets $C_{\zeta}$ which are symmetric with respect to the origin. We take $C_{\zeta}$ to be a strip whose boundary consists of two parallel lines of slope $m$ passing through $\pi i$ and $-\pi i$, respectively.

THEOREM 5.3. Let $\left\{\mathscr{L}_{n}\right\}$ be the sequence of Lidstone functionals, and let $C_{\zeta}=\{\zeta=x+i y \mid m x-\pi<y<m x+\pi\}$ for some real number $m$. A sequence $\left\{b_{n}\right\}$ in $S$ is admissible for $\left\{\mathscr{L}_{n}\right\}$ and $K\left[C_{\zeta}\right]$ if and only if $b(z)$ is regular on the set $1 / C_{\zeta}^{\prime}$ consisting of the closed disks $D_{1}$ and $D_{2}$ having centers at $(1 / 2 \pi)(-m+i)$ and $(1 / 2 \pi)(m-i)$, respectively, and radii $(1 / 2 \pi)\left(m^{2}+1\right)^{1 / 2}$.

Proof. We omit the proof of "only if" since it is almost identical with the proof of Theorem 5.2, and we state the proof of "if" briefly. Let $\left\{b_{n}\right\}$ be such that $b(z)$ is regular on $1 / C_{\zeta}^{\prime}$. Let

$$
H_{1}(z, t)=\frac{\sinh [(1-z) 1 / t]}{\sinh 1 / t} \text { and } H_{2}(z, t)=\frac{1}{t} \frac{\sinh z / t}{\sinh 1 / t}
$$

Define a function $f$ by

$$
f(z)=\frac{1}{2 \pi i} \int_{E} \frac{b(t)}{t}\left[H_{1}(z, t)+H_{2}(z, t)\right] d t
$$


where $E$ is a simple contour symmetric with respect to the origin which encloses $1 / C_{\zeta}^{\prime}$ and is contained in the region of regularity of $b$. Even derivatives of $H_{1}$ and $H_{2}$ with respect to $z$ at $z=0$ and $z=1$ are $H_{1}^{(2 n)}(0, t)=t^{-2 n}$ and $H_{1}^{(2 n)}(1, t)$ $=0 ; H_{2}^{(2 n)}(0, t)=0$ and $H_{2}^{(2 n)}(1, t)=t^{-2 n-1}$; so

$$
\mathscr{L}_{n}(f)=\frac{1}{2 \pi i} \int_{E} \frac{b(t)}{t^{n+1}}=\frac{b^{(n)}(0)}{n !}=b_{n} .
$$

In the same manner as in the proof of Theorem 5.1, we obtain

$$
|f(z)|=O(1) \max _{ \pm \zeta \in \Gamma}\left|e^{z \zeta}\right|
$$

where $\Gamma$ is the image of $E$ under $\zeta=1 / t$, and using the symmetry of $1 / C_{\zeta}^{\prime}$ about the origin,

$$
|f(z)|=O(1) \max _{\zeta \in \Gamma}\left|e^{z \zeta}\right|
$$

so that $D(f)$ is contained in the convex hull of $\Gamma$. Again, since $C_{\zeta}$ is convex, the convex hull of $\Gamma$ is contained in $C_{\zeta}$, so that $D(f) \subseteq C_{\zeta}$, or $f \in K\left[C_{\zeta}\right]$.Q.E.D.

6. Convergence and summability of series. Certain cases of convergence or summability of a series $\sum b_{n} p_{n}(z)$ can be studied by the methods of this paper. If for a given sequence $\left\{\mathscr{L}_{n}\right\}$ of linear functionals having a representation 2.2, there is an associated interpolation series $\sum \mathscr{L}_{n}(f) p_{n}(z)$, Gelfond [6] showed that if $f \in K\left[\Delta_{\zeta}\right]$, then its interpolation series converges for all $z$, and Buck [2] showed that if $f \in K\left[\Omega_{\zeta}^{*}\right]$, the series is Mittag-Leffler summable for all $z$. If for a given sequence $\left\{p_{n}\right\}$ of functions, and a given class $C \subseteq K$ of functions, $\sum b_{n} p_{n}(z)$ is summable by some specified method for all $z$ to a function $f$ in $C$, then $\left\{b_{n}\right\}$ will be said to be admissible for $\left\{p_{n}\right\}$ and $C$. The question of admissibility defined in this way is complicated by the fact that there may be multiple expansions of a function. That is, there may be a sequence $\left\{h_{n}\right\}$ with not all $h_{n}=0$ such that $\sum h_{n} p_{n}(z)=0$. Then if $f(z)=\sum b_{n} p_{n}(z)$, it is also true that $f(z)=$ $\Sigma\left(b_{n}+M h_{n}\right) p_{n}(z)$ for every complex number $M$. Thus, there are infinitely many sequences $\left\{c_{n}\right\}$ all of which are admissible for $\left\{p_{n}(z)\right\}$ and the one function $f$.

We shall restrict the sequences $\left\{p_{n}(z)\right\}$ to be sequences of polynomials which satisfy a formal generating relation

$$
A(w) e^{z g(w)}=\sum_{n=0}^{\infty} p_{n}(z) w^{n}
$$

where $A(w)$ and $g(w)$ are entire with $A(0) \neq 0, g(0)=0$, and $g^{\prime}(0) \neq 0$. Then the polynomials $p_{n}(z)$ are called Sheffer polynomials by Boas and Buck [1]. For example, the polynomial sequences associated with the generalized difference functionals are Sheffer polynomials with $A(w)=1$.

Let $\Omega_{w}$ be an open, simply connected set containing the origin on which $A(w)$ is regular and has only finitely many zeros, and $g(w)$ is regular and univalent. 
Let $\Omega_{\zeta}$ be the image of $\Omega_{w}$ under $\zeta=g(w)$ and let $W(\zeta)$ be the inverse of $g(w)$ defined on $\Omega_{\zeta}$. Let $\Omega_{w}^{*}$ be the star of $\Omega_{w}$ and $\Omega_{\zeta}^{*}$ the image of $\Omega_{w}^{*}$ under the map $g$. Let $\Delta_{w}$ and $\Delta_{\zeta}$ be defined as before with $\rho_{0}$ denoting the radius of $\Delta_{w}$. These sets and functions play the same role as they did previously.

If $A(\alpha)=0$ for some $\alpha(\neq 0)$ in $\Delta_{w}$, then from 6.1, $\sum p_{n}(z) \alpha^{n}=0$ is a nontrivial representation of zero. If $\alpha$ is a zero of $A(w)$ of multiplicity $m$, then $\sum h_{n} p_{n}(z)=0$ for any sequence $\left\{h_{n}\right\}$ such that

$$
h_{n}=\sum_{j=0}^{m-1} M_{j} n(n-1) \cdots(n-j+1) \alpha^{n-j}
$$

for each $n$, where the $M_{j}$ are any complex numbers [1, p. 25].

Let $\left\{p_{n}(z)\right\}$ be a sequence of polynomials with a generating relation 6.1 . Let $C_{w}$ be an open subset of $\Delta_{w}$ containing the origin whose image $C_{\zeta}$ under $\zeta=g(w)$ is convex. Let $\alpha_{1}, \alpha_{2}, \cdots, \alpha_{N}$ be all of the zeros of $A(w)$ in $C_{w}, \alpha_{k}$ being a zero of multiplicity $r_{k}$.

THEOREM 6.3. Under the above conditions, if $\left\{b_{n}\right\}$ in $S$ is such that $b(z)$ is regular on $1 / C_{w}^{\prime}$, except possibly for poles of order at most $r_{k}$ at $\left(\alpha_{k}\right)^{-1}$, $k=1,2, \cdots, N$, then there is a function $f \in K\left[C_{\zeta}\right]$ such that $f(z)=\sum b_{n} p_{n}(z)$ convergent for all $z$.

Proof. Define a function $f$ by

$$
f(z)=\frac{1}{2 \pi i} \int_{E} \frac{b(t)}{t} A\left(\frac{1}{t}\right) e^{z g(1 / t)} d t
$$

where $E$ is a simple contour contained in the region of regularity of $b(z)$ which encloses $1 / C_{w}^{\prime}$, but does not enclose nor pass through any zero of $A(1 / t)$ not contained in $1 / C_{w}^{\prime}$. Then since $E$ is a compact subset of $1 / C_{w}$ and $C_{w} \subseteq \Delta_{w}$,

uniformly on E; so

$$
A\left(\frac{1}{t}\right) e^{z g(1 / t)}=\sum p_{n}(z) \frac{1}{t^{n}}
$$

$$
\begin{aligned}
f(z) & =\frac{1}{2 \pi i} \int_{E} \frac{b(t)}{t}\left(\sum p_{n}(z) \frac{1}{t^{n}}\right) d t \\
& =\sum p_{n}(z) \frac{1}{2 \pi i} \int_{E} \frac{b(t)}{t^{n+1}} d t .
\end{aligned}
$$

For any $n$, the coefficient $(2 \pi i)^{-1} \int_{E} t^{-n-1} b(t) d t$ is the sum of the residues of $t^{-n-1} b(t)$ at the poles $0,1 / \alpha_{1}, 1 / \alpha_{2}, \cdots, 1 / \alpha_{N}$. Since $b(z)=\sum b_{n} t^{n}$ in a neighborhood of the origin, the residue at the origin is $b_{n}$. We shall show that the residue at $\left(\alpha_{k}\right)^{-1}$ is a linear combination of numbers $h_{n}$ such that $\sum h_{n} p_{n}(z)=0$. Let the order of the pole of $t^{-n-1} b(t)$ at $\left(\alpha_{k}\right)^{-1}$ be $m \leqq r_{k}$. Then for some sequence $\left\{B_{n, m+j}\right\}_{j=-m}^{\infty}$ of complex numbers,

$$
t^{-n-1} b(t)=\sum_{j=-m}^{\infty} B_{n, m+j}\left(t-\left(1 / \alpha_{k}\right)\right)^{j}
$$


in some neighborhood of $\left(\alpha_{k}\right)^{-1}$. Then the residue at $\left(\alpha_{k}\right)^{-1}$ is $B_{n, m-1}$. This number will be expressed in terms of $B_{0, i} ; i=0,1, \cdots, m-1$. Let $\phi_{n}(t)=\left[t-\left(\alpha_{k}\right)^{-1}\right]^{m} t^{-n-1} b(t)$; so that $B_{n, m-1}=[(m-1) !]^{-1} \phi^{(m-1)}\left(\left(\alpha_{k}\right)^{-1}\right)$. In 6.4, for $n=0, t^{-1} b(t)$ $=\sum_{j=-m}^{\infty} B_{0 m+j}\left[t-\left(\alpha_{k}\right)^{-1}\right]^{j} ;$ so we have $\phi_{n}(t)=t^{-n} \sum_{j=0}^{\infty} B_{0, j}\left[t-\left(\alpha_{k}\right)^{-1}\right]^{j}$. Then $\phi_{n}^{(m-1)}(t)$

$=\sum_{j=0}^{m-1}(-1) \frac{j(n+j-1) !}{(n-1) !} t^{-n-j} C_{m-1, j} \sum_{i=m-j-1}^{\infty} \frac{i !}{(i-m+j+1) !} B_{0, i}\left(t-\frac{1}{\alpha_{k}}\right)^{i-m+j+1}$.

Evaluating this at $\left(\alpha_{k}\right)^{-1}$, we obtain

$$
\begin{aligned}
B_{n, m-1} & =\frac{1}{(m-1) !} \sum_{j=0}^{m-1}(-1)^{j} \frac{(n+j-1) !}{(n-1) !} \alpha_{k}^{n+j} C_{m-1, j}(m-1-j) ! B_{0, m-j-1} \\
& =\sum_{j=0}^{m-1}(-1)^{j} B_{0, m-j-1}\left(\begin{array}{c}
n+j-1 \\
j
\end{array}\right) \alpha_{k}^{n+j} ;
\end{aligned}
$$

so $B_{n, m-1}=\sum_{j=0}^{m-1} q_{j}(n) \alpha_{k}^{n+j}$ where each $q_{j}$ is a polynomial of degree at most $m-1$. We show that for each $j, h_{n}=q_{j}(n) \alpha_{k}^{n+j}$ have the form 6.2. Since the polynomials $u_{0}(z)=1, u_{i}(z)=z(z-1) \cdots(z-i+1) ; i=1,2, \cdots, m-1$, span the space of all polynomials of degree at most $m-1$, there exist numbers $Q_{i}$ such that $q_{j}(n) \alpha_{k}^{n+j}=\alpha_{k}^{n+j} \sum_{i=0}^{m-1} Q_{i} u_{i}(n)$; so

$$
q_{j}(n) \alpha_{k}^{n+j}=\sum_{i=0}^{m-1} Q_{i} \alpha_{k}^{j+i} u_{i}(n) \alpha_{k}^{n-i}=\sum_{i=0}^{m-1} M_{i, j} n(n-1) \ldots(n-i+1) \alpha_{k}^{n-i} .
$$

Thus $\sum q_{j}(n) \alpha_{k}^{n+j} p_{n}(z)=0$ for each $j$ and $k$. Therefore $f(z)=\Sigma b_{n} p_{n}(z)$.

The proof that $f \in K\left[C_{\zeta}\right]$ is the same as the proof of this in Theorem 3.1. Q.E.D. If $\Omega_{w}^{*}$ is used instead of $\Delta_{w}$, the series is Mittag-Leffler summable to a function $f \in K\left[C_{\eta}\right]$ by the same proof with convergence replaced by Mittag-Leffler summability throughout.

It would be possible to prove a converse of this theorem if we knew that all nontrivial representations of zero arise from zeros of $A(w)$. However, Boas and Buck [1, p. 25] have shown that they may also arise from the nonunivalence of $g(w)$, but whether they may also arise in still other ways is unknown. They did show that if $\Sigma h_{n} p_{n}(z)=0$ for a sequence $\left\{h_{n}\right\}$ such that $h_{n}=O\left(R^{n}\right)$ for some $R<\left|w_{0}\right|$ where $w_{0}$ is the point of $\Omega_{w}^{* \prime}$ nearest the origin, then the $h_{n}$ have the form $6.2[1$, p. 26]. Then, for the special case in which $A(w)$ is entire, and $g(w)$ is entire and is univalent in the whole plane, $\Omega_{w}$ is the whole plane; so all nontrivial representations of zero arise from the zeros of $A(w)$ in $[D(f)]^{\prime}$. In this case, we give a necessary and sufficient condition for admissibility.

Let $\left\{p_{n}(z)\right\}$ be a sequence of Sheffer polynomials for which $A(w)$ is entire and $g(w)$ is entire and is univalent in the whole plane and let $\alpha_{1}, \alpha_{2}, \cdots, \alpha_{N}$ be all of the zeros of $A(w), \alpha_{k}$ being a zero of multiplicity $r_{k}$. Let $C_{\zeta}$ be an open convex 
subset of the $\zeta$-plane containing the origin with image $C_{w}$ in the $w$-plane, and suppose $\alpha_{1}, \alpha_{2}, \cdots, \alpha_{M}$ are all of the zeros of $A(w)$ in $C_{w}$.

THEOREM 6.5. Under the above conditions, a necessary and sufficient condition that a sequence $\left\{b_{n}\right\}$ be admissible for $\left\{p_{n}(z)\right\}$ and $K\left[C_{\zeta}\right]$ is that $b(z)$ be regular on $1 / C_{w}^{\prime}$ except possibly for poles at the points $\left(\alpha_{k}\right)^{-1}$ of order at most $r_{k} ; k=1,2, \cdots, M$.

Proof. Sufficiency follows from Theorem 6.3. To prove necessity, let $B(\zeta)$ $=A(W(\zeta))$. Let $f(z)=\Sigma b_{n} p_{n}(z)$ for all $z$, where $f \in K\left[C_{\zeta}\right]$. Define a function $c(z)$ by

$$
c(z)=\frac{1}{2 \pi i} \int \frac{F(\zeta)}{[1-W(\zeta) z] B(\zeta)} d \zeta
$$

where $\Gamma$ is a simple contour contained in $C_{\zeta}$ enclosing $D(f)$, the origin, and all zeros of $B(\zeta)$ contained in $C_{\zeta}$. Then

$$
\frac{c^{(n)}(0)}{n !}=\frac{1}{2 \pi i} \int_{\Gamma} \frac{[W(\zeta)]^{n}}{B(\zeta)} F(\zeta) d \zeta
$$

which shall be denoted by $c_{n}$, so that $c(z)=\Sigma c_{n} z^{n}$ in some neighborhood of the origin. Since $\Omega_{\zeta}$ is the whole plane, by a theorem of Boas and Buck [1, p. 22], $\sum c_{n} p_{n}(z)$ converges to $f(z)$ for all $z$. From its definition, $c(z)$ is regular for all $z$ such that for all $\zeta$ on $\Gamma, z \neq[W(\zeta)]^{-1}$. Therefore, just as in the proof of Theorem 3.1, $c(z)$ is regular on $1 / C_{w}^{\prime}$. Thus, if $b_{n}=c_{n}$ for all $n$, the conclusion of the theorem holds. Otherwise $\Sigma\left(b_{n}-c_{n}\right) p_{n}(z)$ is a nontrivial representation of zero, and since all of these arise from the zeros $\alpha_{1}, \alpha_{2}, \cdots, \alpha_{M^{\prime}}$ of $A(w)$ in $[D(f)]^{\prime}$, we have

$$
h_{n}=b_{n}-c_{n}=\sum_{k=1}^{M^{\prime}} \sum_{j=0}^{r_{k}-1} M_{k j} n(n-1) \cdots(n-j+1) \alpha_{k}^{n-j} .
$$

Then, for $h(z)=\Sigma h_{n} z^{n}$,

$$
\begin{aligned}
h(z) & =\sum_{n=0}^{\infty} \sum_{k=1}^{M^{\prime}} \sum_{j=0}^{r_{k}-1} M_{k j} n(n-1) \cdots(n-j+1) \alpha_{k}^{n-j} z^{n} \\
& =\sum_{k=1}^{M^{\prime}} \sum_{j=0}^{r_{k}-1} M_{k j} \sum_{n=j}^{\infty} n(n-1) \cdots(n-j+1) \alpha_{k}^{n-j} z^{n} \\
& =\sum_{k=1}^{M^{\prime}} \sum_{j=0}^{r_{k}-1} M_{k j} \frac{d^{j}}{d \alpha_{k}^{j}}\left(\sum_{n=0}^{\infty} \alpha_{k}^{n} z^{n}\right) \\
& =\sum_{k=1}^{M^{\prime}} \sum_{j=0}^{r_{k}-1} \frac{M_{k j} j ! z^{j}}{\left(1-\alpha_{k} z\right)^{j}} .
\end{aligned}
$$

Thus $b(z)=c(z)+h(z)$ where $c(z)$ is regular on $1 / C_{w}^{\prime}$ and $h(z)$ is rational with poles of order at most $r_{k}$ at $1 / \alpha_{k}, k=1,2, \cdots, M^{\prime}$, so $b(z)$ is regular on $1 / C_{w}^{\prime}$ except possibly for poles of order at most $r_{k}$ at $\left(\alpha_{k}\right)^{-1} ; k=1,2, \cdots, M$. Q. E. D. 
If the polynomials $p_{n}(z)$ satisfy

$$
A(w) e^{z w}=\sum_{n=0}^{\infty} p_{n}(z) w^{n}
$$

where $A(w)$ is a polynomial, then the ordinary linear differential equation

$$
A\left(\frac{d}{d z}\right) y=f(z)
$$

where $f \in K$, has solutions $y(z)=\sum c_{n} z^{n} / n$ ! for every sequence $\left\{c_{n}\right\}$ such that $f(z)=\sum c_{n} p_{n}(z)[1$, p. 68]. Theorem 6.5 then implies that the conjugate indicator diagram $D(y)$ for any solution $y(z)$ is contained in the convex hull of the union of $D(f)$ with the zeros $\alpha_{1}, \alpha_{2}, \cdots, \alpha_{M}$ of $A(w)$ in $[D(f)]^{\prime}$. Actually, since $b(z)$ $=c(z)+h(z)$ where $h(z)$ is given by 6.6 , the solutions are given by

$$
\begin{aligned}
y(z) & =\sum_{n=0}^{\infty} c_{n} z^{n} / n !+\sum_{k=1}^{M^{\prime}} \sum_{j=0}^{r_{k}-1} M_{k j} \frac{d^{j}}{d \alpha_{k}^{j}}\left(\sum_{n=0}^{\infty} \alpha_{k}^{n} z^{n} / n !\right) \\
& =\sum_{n=0}^{\infty} c_{n} z^{n} / n !+\sum_{k=1}^{M^{\prime}} \sum_{j=0}^{r_{k}-1} M_{k j} \frac{d^{j}}{d \alpha_{k}^{j}} e^{\alpha_{k} z} \\
& =y^{*}(z)+\sum_{k=1}^{M^{\prime}} \sum_{j=0}^{r_{k}-1} M_{k j} z^{j} e^{\alpha_{k} z}
\end{aligned}
$$

where $D\left(y^{*}\right)=D(f)$.

\section{BIBLIOGRAPHY}

1. R. P. Boas and R. C. Buck, Polynomial expansions of analytic functions, Springer-Verlag, Berlin, 1958.

2. R. C. Buck, Interpolation series, Trans. Amer. Math. Soc. 64 (1948), 283-298.

3. - Integral valued entire functions, Duke Math. J. 15 (1948), 879-891.

4. - On admissibility of sequences and a theorem of Polya, Comment. Math. Helv. 27 (1953), 75-80.

5. - On n-point expansions of entire functions, Proc. Amer. Math. Soc. 6 (1955). 793-796.

6. A. Gelfond, Interpolation et unicité des fonctions entieres, Mat. Sb. (N.S.) 46 (1938), 115-147.

7. G. Pólya, Uber Lucken und Singularitaten von Potenzreihen, Math. Z. 29 (1929), 549-640.

MiAMI UNIVERSITY,

OXFORD, OHIO 